\title{
Generalized Multiscale Approximation of Mixed Finite Elements with Velocity Elimination for Subsurface Flow
}

\author{
Jie Chen ${ }^{\mathrm{a}, \mathrm{c}}$, Eric T. Chung ${ }^{\mathrm{b}}$, Zhengkang He ${ }^{\mathrm{c}}$, Shuyu Sun ${ }^{\mathrm{d}, *}$ \\ ${ }^{a}$ Department of Mathematical Sciences, Xian Jiaotong-Liverpool University, Suzhou, PR China 215123 \\ ${ }^{b}$ Department of Mathematics, The Chinese University of Hong Kong (CUHK), Hong Kong SAR \\ ${ }^{c}$ School of Mathematics and Statistics, Xi'an Jiaotong University, Xi'an, P.R.China 710049 \\ ${ }^{d}$ Computational Transport Phenomena Laboratory, Division of Physical Science and Engineering, King \\ Abdullah University of Science and Technology, Thuwal 23955-6900, Kingdom of Saudi Arabia
}

\begin{abstract}
A frame work of the mixed generalized multiscale finite element method (GMsFEM) for solving Darcy's law in heterogeneous media is studied in this paper. Our approach approximates pressure in multiscale function space that is between fine-grid space and coarse-grid space and solves velocity directly in the fine-grid space. To construct multiscale basis functions for each coarse-grid element, three types of snapshot space are raised. The first one is taken as the fine-grid space for pressure and the other two cases need to solve a local problem on each coarse-grid element. We describe a spectral decomposition in the snapshot space motivated by the analysis to further reduce the dimension of the space that is used to approximate the pressure. Since the velocity is directly solved in the fine-grid space, in the linear system for the mixed finite elements, the velocity matrix can be approximated by a diagonal matrix without losing any accuracy. Thus it can be inverted easily. This reduces computational cost greatly and makes our scheme simple and easy for application. Comparing to our previous work of mixed generalized multiscale finite element method [E. T. Chung, Y. Efendiev, and C. S. Lee. Mixed generalized multiscale finite element methods and applications. Multiscale Modeling and Simulation, 13(1):338-366, 2015.], both the pressure and velocity space in this approach are bigger. As a consequence, this method enjoys better accuracy. While the computational cost does not increase because of the good property of velocity matrix. Moreover, the proposed method preserves the local mass conservation property that is important for subsurface problems. Numerical examples are presented to illustrate the good properties of the proposed approach. If offline spaces are appro-

\footnotetext{
* Corresponding author: Shuyu Sun.

Email addresses: jie.chenolexjtlu.edu.cn (Jie Chen), tschung@math.cuhk.edu.hk (Eric T. Chung), hzk2abc@163. com (Zhengkang He), shuyu. sun@kaust.edu. sa (Shuyu Sun)

${ }^{1}$ The work is supported by the National Natural Science Foundation of China (No.11401467 and No.51874262), Introduction plan of senior foreign experts (G20190027012), and XJTLU Key Programme Special Fund (KSF-P-02). The work is also supported in part by funding from King Abdullah University of Science and Technology (KAUST) through the grant BAS/1/1351-01. The research of Eric Chung is supported by Hong Kong RGC General Research Fund (project 14317516) and CUHK Direct Grant for Research 2017-18.
} 
priately selected, one can achieve good accuracy with only a few basis functions per coarse element according to the numerical results.

Keywords: multiscale, porous media, mixed finite elements, local mass conservation, two-phase flow

\section{Introduction}

Multiscale phenomena extensively exist in subsurface flows. The subsurface property permeability can span multiple scales and be of several orders of magnitude. For example, in the fractured and shale reservoir, the permeability in the fractures and shale layers is much bigger than the one in the matrix. And the thicknesses of these layers are much smaller than the domain size. Moreover, these subsurface flow problems often need to be solved repeatedly with different boundary conditions and source terms. As a consequence, the model reduction technique that can reduce the degrees of freedom significantly is required by such subsurface problems. A traditional approach is to solve local problems in each coarse block and to compute the effective permeability $[18,11,37]$. This method can be extended to multi-phase flow [28, 38, 35, 39] by upscaling relative permeabilities $[6,19]$. An alternative method to reduce the model is the multiscale method [25, 22, 26, 21, 13, 27], in which the local solutions on a coarse element are employed as basis functions in the simulation. Since local mass conservation is important for subsurface problems, mixed multiscale finite element methods $[12,1,2,3,9,10,16]$, multiscale finite volume methods [27, 24, 30, 17, 29], mortar multiscale methods $[33,4,32,15]$, and various post-processing approaches are proposed to obtain mass conservative velocity fields $[8,31]$.

The mixed multiscale finite element method is a widely used mass conservative multiscale approach $[12,1,2,3]$, in which the multiscale technique is mainly used for velocity. Multiscale basis functions for the velocity field are found by solving a local problem with Neumann boundary conditions with support to be two neighbouring coarse elements sharing a common edge. Piecewise constant basis functions, whose support is a single coarse element, are used for the pressure equations. In these methods, spatially distributed fluxes are imposed on internal edges to reduce the subgrid effects and for each edge, only one multiscale basis function is employed. Recently, a mixed generalized multiscale finite element method has been raised to solve the multiscale and high-contrast problems $[14,23,13,20]$. The main advantage of the mixed GMsFEM is that multiple multiscale basis functions for the velocity can be used in two coarse blocks sharing a common edge. To compute the multiple velocity basis functions, local cell problems are solved in two coarse-block regions to construct the snapshot spaces and then the snapshot spaces are further reduced by solving local spectral problems designed according to the analysis. Typical local cell problems consist of the same governing equations as the original problem together with prescribed Neumann boundary conditions on the common edge between two coarse blocks. Ordering the eigenvalues in increasing order, the eigenvectors corresponding to small eigenvalues are selected to construct offline basis functions. The convergence analysis of these methods gives a spectral convergence $1 / \Lambda$, where $\Lambda$ is the smallest eigenvalue corresponding to the unused eigenvector [14]. 
In this paper, a frame work of the mixed generalized multiscale finite element method (GMsFEM) for subsurface problems is proposed. Different from the previous approaches, multiple multiscale basis functions for the pressure are computed in single coarse element and the velocity is approximated in the fine grid space. To construct multiscale basis functions for each coarse-grid element, three types of snapshot space are raised. The first one is taken as the fine-grid space for pressure and the other two cases need to solve a local problem on each coarse-grid element. We describe a spectral decomposition in the snapshot space motivated by the analysis to further reduce the dimension of the space that is used to approximate the pressure. Although the velocity is directly solved on fine mesh, with the help of trapezoidal quadrature rule, the stiffness matrix for velocity is diagonal and can be inverted easily. Thus we only need to solve a linear system in multiscale finite element space for pressure. If offline spaces are appropriately selected, one can achieve good accuracy with only a few basis functions per coarse element according to our numerical results.

The rest of this paper is arranged as follows. Section 2 introduces the basic model of subsurface flows, the definition of notations and fine-scale discretization. Section 3 illustrates a systematic way to construct the snapshot space and offline space. A convergence analysis for the proposed mixed generalized multiscale finite element method is presented in section 4. Numerical results and discussions are shown in section 5. The paper ends with a conclusion.

\section{Preliminaries}

We consider the following flow problem in mixed formulation with high-contrast coefficient:

$$
\begin{aligned}
k^{-1} \mathbf{u}+\nabla p=0 \quad \text { in } \quad \Omega, \\
\nabla \cdot \mathbf{u}=f \quad \text { in } \quad \Omega,
\end{aligned}
$$

with nonhomogeneous boundary condition

$$
\mathbf{u} \cdot \mathbf{n}=g \quad \text { on } \quad \partial \Omega,
$$

where $k$ is a high-contrast heterogeneous permeability, $\mathbf{u}$ is the Darcy velocity, $p$ is the pressure, $f$ is the sourece term, $g$ is the given normal component of Darcy velocity on the boundary, $\Omega$ is the computational domain and $\mathbf{n}$ is the outward unit norm vector on the boundary.

To illustrate the general solution framework of the proposed mixed GMsFEM, the notations of coarse and fine grids are introduced as follows. Let $\mathcal{T}^{H}$ be a conforming partition of the computational domain $\Omega$ into finite elements (quadrilaterals, parallelepipeds, trangles, tetrahedrals, etc.)with coarse-block size $H$ and $\mathcal{T}^{h}$ be the fine-grid partition with mesh size $h$. We use $\mathcal{E}^{H}:=\bigcup_{i=1}^{N_{e}} E_{i}$ to denote the set of all edges in the coarse mesh $\mathcal{T}^{H}$, where $N_{e}$ is the number of coarse edges. We also define the element in coarse mesh as $T_{i}$, thus $\Omega:=\bigcup_{i=1}^{N_{t}} T_{i}$ ( $N_{t}$ is the number of elements in the coarse mesh). The element in the fine mesh is defined as $t_{i}$, thus $\Omega:=\bigcup_{i=1}^{M_{t}} t_{i}$ with $M_{t}$ being 
the number of elements in the fine mesh. The set of edges in the fine grid is denoted by $\mathcal{E}^{h}:=\bigcup_{i=1}^{M_{e}} e_{i}$, where $M_{e}$ is the number of fine edges.

Define

$$
L^{2}(\Omega)=\left\{v: v \text { is defined on } \Omega \text { and } \int_{\Omega} v^{2} \mathrm{~d} x<\infty\right\}
$$

We also use the space

$$
\mathbf{H}(\operatorname{div}, \Omega)=\left\{\mathbf{v}=\left(v_{1}, v_{2}\right) \in\left(L^{2}(\Omega)\right)^{2}: \nabla \cdot \mathbf{v} \in L^{2}(\Omega)\right\} .
$$

Define

$$
\mathbf{V}=\mathbf{H}(\operatorname{div}, \Omega), \quad W=L^{2}(\Omega)
$$

Recall $\mathcal{T}^{h}$ is the fine partition of $\Omega$ into non-overlapping (open) elements. Define the mixed finite element spaces (we take the mixed finite element spaces on quadrilaterals for illustration).

$$
\begin{aligned}
\mathbf{V}_{h} & =\left\{\mathbf{v}_{h} \in \mathbf{V}:\left.\mathbf{v}_{h}\right|_{t}=\left(b_{t} x_{1}+a_{t}, d_{t} x_{2}+c_{t}\right), a_{t}, b_{t}, c_{t}, d_{t} \in \mathbb{R}, t \in \mathcal{T}^{h}\right\} \\
W_{h} & =\left\{w_{h} \in W: w_{h} \text { is a constant on each element in } \mathcal{T}^{h}\right\} .
\end{aligned}
$$

The normal components of $\mathbf{v}_{h} \in \mathbf{V}_{h}$ are continuous across the interior edges in $\mathcal{T}^{h}$. Thus, the fine-grid solution $\left(\mathbf{u}_{h}, p_{h}\right) \in\left(\mathbf{V}_{h}, W_{h}\right)$ satisfies

$$
\begin{array}{rlrl}
\int_{\Omega} k^{-1} \mathbf{u}_{h} \cdot \mathbf{v}_{h}-\int_{\Omega} \operatorname{div}\left(\mathbf{v}_{h}\right) p_{h} & =0 & & \forall \mathbf{v}_{h} \in \mathbf{V}_{h}^{0}, \\
\int_{\Omega} \operatorname{div}\left(\mathbf{u}_{h}\right) w_{h} & =\int_{\Omega} f w_{h} & \forall w_{h} \in W_{h}
\end{array}
$$

where $\mathbf{u}_{h} \cdot \mathbf{n}=g_{h}$ on $\partial \Omega$ with $g_{h}$ being the average of function $g$ on each fine boundary edge and $\mathbf{V}_{h}^{0}=\left\{\mathbf{v}_{h} \in \mathbf{V}_{h}: \mathbf{v}_{h} \cdot \mathbf{n}=0\right.$ on $\left.\partial \Omega\right\}$. The above system can be recast in matrix form

$$
\begin{aligned}
M_{\text {fine }} \mathbf{U}_{h}+B_{\text {fine }} \mathbf{P}_{h} & =\mathbf{0}, \\
B_{\text {fine }} \mathbf{U}_{h} & =\mathbf{F}_{h} .
\end{aligned}
$$

The velocity will be approximated directly in the fine-grid space $\mathbf{V}_{h}$. The approximation of the pressure will be found in the multisale finite element space which is between the coarse-grid space and fine-grid space. A set of multiscale basis functions for the pressure are defined in each coarse-grid element with the support to be the corresponding coarse element. Let $\left\{\Psi_{j}\right\}$ be the set of multiscale basis functions for the coarse element $T_{i}$. The multiscale space for the pressure $p$ is defined as the linear span of all local basis functions, which can be denoted as

$$
W_{H}=\bigoplus_{\mathcal{T}^{H}}\left\{\Psi_{i}\right\} .
$$


The mixed GMsFEM is to find $\left(\mathbf{u}_{H}, p_{H}\right) \in\left(\mathbf{V}_{h}, W_{H}\right)$ such that

$$
\begin{array}{rlrl}
\int_{\Omega} k^{-1} \mathbf{u}_{H} \cdot \mathbf{v}_{H}-\int_{\Omega} \operatorname{div}\left(\mathbf{v}_{H}\right) p_{H} & =0 & & \forall \mathbf{v}_{H} \in \mathbf{V}_{h}^{0}, \\
\int_{\Omega} \operatorname{div}\left(\mathbf{u}_{H}\right) w_{H} & =\int_{\Omega} f w_{H} & \forall w_{H} \in W_{H},
\end{array}
$$

where $\mathbf{u}_{H} \cdot \mathbf{n}=g_{H}$ on $\partial \Omega$ and for each coarse edge on the boundary and $g_{H}$ is the average of function $g$ on the corresponding coarse edge.

\section{The construction of multiscale basis functions}

In this section, we will illustrate a systematic way to construct the multiscale space $W_{H}$ for approximating the pressure $p$. The constructions of snapshot spaces are firstly introduced. There are three possible ways to do this. The first case simply takes the fine-grid space for pressure $W_{h}$ to be the snapshot space. The other two contain an extensive set of basis functions formed by the solutions of local problems with Dirichlet and Neumann boundary conditions, respectively. Then the snapshot space is further reduced by solving a local spectral problem to obtain the dominant modes. The spectral problem is carefully designed to reduce the residual rapidly. The linear span of the obtained dominant modes is called the offline space. Since the problem we considered is parameter independent, the offline space is the same as the online space.

\subsection{Snapshot space}

There are three ways to construct the snapshot space. We illustrate them one by one.

Case I: The snapshot space $W_{\text {snap }}$ can be simply taken as the fine-grid space for the pressure $W_{h}$. That is

$$
W_{\text {snap }}=\left\{\Psi_{i}^{\text {snap }} \in W: \Psi_{i}^{\text {snap }} \text { is a constant on each element } t_{i} \text { in } \mathcal{T}^{h}\right\} .
$$

Case II: Let $T_{i} \in \mathcal{T}^{H}$ be a coarse element in $\Omega$. We will find $\left(\mathbf{u}_{j}^{(i)}, p_{j}^{(i)}\right) \in$ $\left.\left(\mathbf{V}_{h}, W_{h}\right)\right|_{T_{i}}$ by solving the following problem on the coarse element $T_{i}$ :

$$
\begin{aligned}
k^{-1} \mathbf{u}_{j}^{(i)}+\nabla p_{j}^{(i)}=0 & \text { in } T_{i}, \\
\operatorname{div}\left(\mathbf{u}_{j}^{(i)}\right)=0 & \text { in } T_{i},
\end{aligned}
$$

where $\left.\left(\mathbf{V}_{h}, W_{h}\right)\right|_{T_{i}}$ is the restrition of $\left(\mathbf{V}_{h}, W_{h}\right)$ on the coarse-grid element $T_{i}$. Notice that the boundary of coarse-grid element can be expressed as a union of fine-grid edges, i.e., $\partial T_{i}=\bigcup_{j=1}^{J_{i}} e_{j}$, where $J_{i}$ is the total number of fine-grid edges on the boundary of coarse-grid element $T_{i}$. Let $\delta_{j}^{(i)}$ be a piecewise constant function defined on $\partial T_{i}$ with respect to the fine grid such that it has value 1 on $e_{j}$ and value 0 on the other fine-grid 
edges; That is,

$$
\delta_{j}^{(i)}= \begin{cases}1 & \text { in } e_{j}, \\ 0 & \text { on other fine-grid edges on } \partial T_{i}, \quad j=1,2, \cdots, J_{i} .\end{cases}
$$

Thus the boundary condition on the boundary of coarse-grid element $T_{i}$ for the local problem (3.1) is taken as

$$
p_{j}^{(i)}=\delta_{j}^{(i)} \quad \text { on } \partial T_{i} .
$$

The above local problem (3.1) together with the Dirichlet boundary condition (3.3) can be solved numerically on fine grid $T_{i}$ by the lowest-order Raviart-Thomas elements, so that the resulting pressure $p_{j}^{(i)} \in W_{h}$ (we keep the same notation for the discrete solution $p_{j}^{(i)}$ for simplicity).

Case III: Let $T_{i} \in \mathcal{T}^{H}$ be a coarse element in $\Omega$. We will find $\left(\mathbf{u}_{j}^{(i)}, p_{j}^{(i)}\right) \in$ $\left.\left(\mathbf{V}_{h}, W_{h}\right)\right|_{T_{i}}$ by solving the following problem on the coarse element $T_{i}$ :

$$
\begin{aligned}
k^{-1} \mathbf{u}_{j}^{(i)}+\nabla p_{j}^{(i)} & =0 & & \text { in } T_{i}, \\
\operatorname{div}\left(\mathbf{u}_{j}^{(i)}\right) & =\alpha_{j}^{(i)} & & \text { in } T_{i},
\end{aligned}
$$

together with the Neumann boundary condition on the boundary of coarse-grid element $T_{i}$

$$
\frac{\partial p_{j}^{(i)}}{\partial \mathbf{n}_{i}}=\delta_{j}^{(i)} \quad \text { on } \partial T_{i},
$$

where $\mathbf{n}_{i}$ is an outward unit norm vector on $\partial T_{i}$ and $\alpha_{j}^{(i)}$ is chosen so that the compatibility condition $\int_{T_{i}} \alpha_{j}^{(i)}=\int_{\partial T_{i}} \delta_{j}^{(i)}$ is satisfied. In this case, $p$ is unique up to an additive constant. Similarly, the above local problem (3.4) together with the Neumann boundary condition (3.5) can be solved numerically on fine grid $T_{i}$ by the lowest-order Raviart-Thomas elements, so that the resulting pressure $p_{j}^{(i)} \in W_{h}$. We remark that the velocity satisfies $\mathbf{u} \cdot \mathbf{m}_{i}=\delta_{j}^{(i)}$ on $\partial T_{i}$ in this case.

The collection of the solutions of above local problems (Case II or Case III) generates the snapshot space. We let $\Psi_{j}^{i, \text { snap }}:=p_{j}^{(i)}$ be the snapshot fields and define the snapshot space $W_{\text {snap }}$ by

$$
W_{\text {snap }}=\operatorname{span}\left\{\Psi_{j}^{i, \text { snap }}: 1 \leq j \leq J_{i}, 1 \leq i \leq N_{t}\right\}
$$

To simplify expressions, we use the single-index notation

$$
W_{\text {snap }}=\operatorname{span}\left\{\Psi_{i}^{\text {snap }}: 1 \leq i \leq M_{\text {snap }}\right\},
$$

where $M_{\text {snap }}=\sum_{i=1}^{N_{t}} J_{i}$ is the total number of snapshot fields.

Remark 3.1. For Case I, there is no extra computational cost for the local problem 
and the dimension of the snapshot space in coarse element $T_{i}$ is the number of fine elements contained in $T_{i}$. For Case II and III, we need to solve local problems in each coarse-grid element and the dimension of the snapshot space is reduced to the number of fine edges $e_{j}$ on the boundary of coarse element $T_{i}$.

Remark 3.2. For the boundary element that has two or more boundary edges (for example corner elements), all the edges belong to this element are considered as a single boundary edge for the definition of the function $\delta_{j}^{(i)}$, so that all $\Psi_{i}^{\text {snap }}$ in the snapshot space $W_{\text {snap }}$ are linearly independent.

Remark 3.3. For Case III, since the Neumann boundary condition for pressure is used in the local problem, the solution $p$ is unique up to an additive constant. As a consequence, we emphasize that the piecewise constant basis on the coarse-grid element should be included in the snapshot space $W_{\text {snap. }}$

Each multiscale basis function $\Psi_{i}^{\text {snap }}$ can be represented as a linear combination of fine-grid basis functions in $W_{h}$. Thus, we can use the coefficients vector $\psi_{i}^{\text {snap }}$ to represent the multiscale basis function $\Psi_{i}^{\text {snap }}$. We define

$$
R_{\text {snap }}=\left[\psi_{1}^{\text {snap }}, \cdots, \psi_{M_{\text {snap }}}^{\text {snap }}\right]
$$

which maps from the snapshot space to the fine space. Specially, for Case I, $R_{\mathrm{snap}}$ is an identity matrix.

\subsection{Offline space}

In this subsection, we further reduce the snapshot space by solving some local spectral problems and we term the solutions of local spectral problems the offline space. The offline space contains the important modes in the snapshot space and can approximate the solution efficiently. We consider the spectral problem of finding a real number $\lambda$ and a function $p \in W_{\text {snap }}$ such that

$$
a(p, w)=\lambda s(p, w), \quad \forall w \in W_{\text {snap }},
$$

where $a(p, w)$ and $s(p, w)$ are positive semidefinite symmetric bilinear forms defined on the snapshot space $W_{\text {snap }} \times W_{\text {snap }}$.

It is inefficient to solve the global spectral problem (3.6). Thus we solve it in each coarse-grid element $T_{i}$. We let $W_{\text {snap }}^{(i)}$ be the snapshot space corresponding to the coarse element $T_{i}$, which is defined by

$$
W_{\text {snap }}^{(i)}=\operatorname{span}\left\{\Psi_{j}^{i, \text { snap }}: 1 \leq j \leq J_{i}\right\} .
$$

The following is the local spectral problem: Find a real number $\lambda \geq 0$ and a function $p \in W_{\text {snap }}^{(i)}$ such that

$$
a_{i}(p, w)=\lambda s_{i}(p, w) \quad \forall w \in W_{\text {snap }}^{(i)} .
$$


For each coarse element $T_{i}$, we take

$$
a_{i}(p, w)=\sum_{e} k[p][w], \quad s_{i}(p, w)=\int_{T_{i}} k p w,
$$

where $[p]$ and $[w]$ are the jump of functions $p$ and $w$, respectively. $e$ is an interior fine edge in coarse-grid element $T_{i}$.

Remark 3.4. The definition of spectral problem (3.7) and (3.8) is suitable for all the three cases of the snapshot space. For case II and III, where a local problem is solved, the spectral problem (3.7) and (3.8) is equivalent to

$$
a_{i}(p, w)=\lambda s_{i}(p, w) \quad \forall w \in W_{\text {snap }}^{(i)} .
$$

where

$$
a_{i}(p, w)=\int_{T_{i}} k \nabla p \cdot \nabla w=\int_{T_{i}} k^{-1} \mathbf{u} \cdot \mathbf{v}, \quad s_{i}(p, w)=\int_{T_{i}} k p w .
$$

We arrange the eigenvalues of (3.7) in increasing order,

$$
\lambda_{1}^{(i)}<\lambda_{2}^{(i)}<\cdots<\lambda_{J_{i}}^{(i)},
$$

where $\lambda_{k}^{(i)}$ denotes the $k$ th eigenvalue for the coarse-grid element $T_{i}$. The corresponding eigenvector is expressed as $Z_{k}^{(i)}=\left(Z_{k j}^{(i)}\right)_{j=1}^{J_{i}}$ with $Z_{k j}^{(i)}$ being the $j$ th component of the vector $Z_{k}^{(i)}$. The first $l_{i}$ eigenfunctions are selected to form the offline space. Using the eigenfunctions, the offline basis functions can be constructed as

$$
\Psi_{k}^{i, \text { off }}=\sum_{j=1}^{J_{i}} Z_{k j}^{(i)} \Psi_{j}^{i \text {,snap }}, \quad k=1,2, \cdots, l_{i} .
$$

Then the global offline space is

$$
W_{\text {off }}=\operatorname{span}\left\{\Psi_{k}^{i, \text { off }}: 1 \leq k \leq l_{i}, 1 \leq i \leq N_{t}\right\}
$$

Using the single-index notation to simplify expression, we get

$$
W_{\text {off }}=\operatorname{span}\left\{\Psi_{k}^{\text {off }}: 1 \leq k \leq M_{\text {off }}\right\}
$$

where $M_{\text {off }}=\sum_{i=1}^{N_{t}} l_{i}$ is the total number of offline basis functions. We will employ this space to approximate the pressure, i.e. $W_{H}=W_{\text {off }}$ in the mixed GMsFEM system (2.5). The above eigenvalue problem (3.6) can be recast in matrix form

$$
A_{\text {snap }}^{(i)} Z_{k}^{(i)}=\lambda_{k}^{(i)} S_{\text {snap }}^{(i)} Z_{k}^{(i)},
$$


where

$$
\begin{aligned}
& A_{\text {snap }}^{(i)}=a_{i}\left(\Psi_{m}^{i, \text { snap }}, \Psi_{n}^{i, \text { snap }}\right)=R_{\text {snap }}^{T} A_{\text {fine }}^{(i)} R_{\text {snap }} \\
& S_{\text {snap }}^{(i)}=s_{i}\left(\Psi_{m}^{i, \text { snap }}, \Psi_{n}^{i, \text { snap }}\right)=R_{\text {snap }}^{T} S_{\text {fine }}^{(i)} R_{\text {snap }} .
\end{aligned}
$$

The notations $A_{\text {snap }}^{(i)}$ and $S_{\text {snap }}^{(i)}$ express the fine-scale matrices using fine-grid basis functions in coarse element $T_{i}$. Each $\Psi_{k}^{\text {off }}$ can be expressed by a vector $\psi_{k}^{\text {off }}$ containing the coefficients in the expansion of $\Psi_{k}^{\text {off }}$ in the fine-grid basis functions. Thus we have

$$
R_{\mathrm{off}}=\left[\psi_{1}^{\mathrm{off}}, \cdots, \psi_{M_{\mathrm{off}}}^{\mathrm{off}}\right]
$$

which maps from the offline space to the fine space. In terms of matrix expressions, the mixed GMsFEM system (2.5) can be represented as

$$
\begin{aligned}
M_{\text {fine }} \mathbf{U}_{H}+B_{\text {fine }}^{T} R_{\text {off }} \mathbf{P}_{H} & =0, \\
R_{\text {off }}^{T} B_{\text {fine }} \mathbf{U}_{H} & =R_{\text {off }}^{T} \mathbf{F}_{H},
\end{aligned}
$$

where the matrix $M_{\text {fine }}$ is symmetric, positive definite, and sparse. $\mathbf{U}_{H}$ and $P_{H}$ are unknown velocity and pressure vectors in the spaces $\mathbf{V}_{h}$ and $W_{H}$, respectively. As a consequence, to implement the proposed mixed GMsFEM, we need to construct the fine-grid matrices $M_{\text {fine, }}, B_{\text {fine }}$ and the offline matrix $R_{\text {off }}$.

Since the velocity is directly solved on the fine mesh, in the case that $k$ is a diagonal tensor, the matrix $M_{\text {fine }}$ can be approximated by a diagonal matrix $\widehat{M_{\text {fine }}}$ when the trapezoidal quadrature rule is used [34, 5]. The lowest order Raviart-Thomas mixed finite element method is equivalent to a finite difference scheme with a five-point stencil in two dimensional space and the modified scheme has the same convergence rate as the unmodified scheme. In other words, we can replace $M_{\text {fine }}$ with a diagonal matrix $\widehat{M_{\text {fine }}}$ without losing any accuracy. Thus $\widehat{M_{\text {fine }}}$ is easy to invert and we can solve the system (3.11) in following way

$$
-R_{\text {off }}^{T} B_{\text {fine }}{\widehat{M_{\text {fine }}}}^{-1} B_{\text {fine }}^{T} R_{\text {off }} \mathbf{P}_{H}=R_{\text {off }}^{T} \mathbf{F}_{H} .
$$

In this way, the original mixed formulation is approximated by a positive definite and sparse linear system for the pressure unknowns and there are only several pressure unknowns per coarse element. As a consequence, the modified system is substantially smaller and easier to solve than the hybrid system used in [14].

\section{Convergence of the mixed GMsFEM}

The convergence analysis of the proposed mixed GMsFEM is presented in this section. We first introduce a projection of the fine-grid velocity field $\mathbf{u}_{h}$ by solving a carefully designed local problem and then derive an error estimate between this projection and fine-grid solution. Next, An estimate between the mixed GMsFEM solution 
and the projection is derived. Combining these two estimations, we have an estimate between the mixed GMsFEM and fine-grid solutions.

Recall that $\left(\mathbf{u}_{h}, p_{h}\right) \in \mathbf{V}_{h} \times W_{h}$ is the fine-grid solution obtained in (2.3). We will define a projection $\widehat{\mathbf{u}} \in \mathbf{V}_{h}$ as follows. Let $T$ be a coarse-grid block and let $\bar{f}$ be $L^{2}$ projection of the source term $f$ onto the space $W_{H}$

$$
\int_{T}(f-\bar{f}) w=0, \quad w \in W_{H} .
$$

Then, the restriction of $\widehat{\mathbf{u}}$ on $T$ is obtained by solving the following problem

$$
\begin{aligned}
k^{-1} \widehat{\mathbf{u}}+\nabla \widehat{p} & =0 \quad \text { in } \quad T, \\
\operatorname{div}(\widehat{\mathbf{u}})=\bar{f} & \text { in } \quad T,
\end{aligned}
$$

subject to the following conditions

$$
\widehat{\mathbf{u}} \cdot \mathbf{n}=\mathbf{u}_{h} \cdot \mathbf{n}, \text { on } \partial T \text { and } \int_{T} \widehat{p}=\int_{T} p_{h} .
$$

The above problem (4.1)-(4.2) is solved on the fine grid, thus we have $\widehat{\mathbf{u}} \in \mathbf{V}_{h}$.

We introduce a weighted $L^{2}$ norm that will be used in the following analysis. Let $T$ be an open set and $\mathbf{u}$ be a vector field. Then the weighted $L^{2}$ norm is defined as $\|\mathbf{u}\|_{k^{-1}, T}=\left(\int_{T} k^{-1}|\mathbf{u}|^{2}\right)^{\frac{1}{2}}$.

Next, we prove the following estimate for $\widehat{\mathbf{u}}$.

Lemma 4.1. Let $\left(\mathbf{u}_{h}, p_{h}\right) \in \mathbf{V}_{h} \times W_{h}$ be the fine-grid solution obtained in (2.3) and $\widehat{\mathbf{u}} \in \mathbf{V}_{h}$ be the solution of (4.1)-(4.2). We have

$$
\int_{\Omega} k^{-1}\left|\mathbf{u}_{h}-\widehat{\mathbf{u}}\right|^{2}+\int_{\Omega}\left(\operatorname{div}\left(\mathbf{u}_{h}-\widehat{\mathbf{u}}\right)\right)^{2} \preceq \max _{T \in \mathcal{T}^{H}}\left(k_{\min , T}^{-1}\right) \sum_{i=1}^{N_{t}}\|f-\bar{f}\|_{L^{2}\left(T_{i}\right)}^{2},
$$

where $k_{\min , T}$ is the minimum of $k$ over $T$.

Proof. Let $T \in \mathcal{T}^{H}$ be a given coarse-grid element. First, substracting (2.3) by the variational form of (4.1), we get

$$
\begin{array}{r}
\int_{T} k^{-1}\left(\mathbf{u}_{h}-\widehat{\mathbf{u}}\right) \cdot \mathbf{v}_{h}-\int_{T} \operatorname{div}\left(\mathbf{v}_{h}\right)\left(p_{h}-\widehat{p}\right)=0, \quad \forall \mathbf{v}_{h} \in \mathbf{V}_{h}^{0}(T), \\
\int_{T} \operatorname{div}\left(\mathbf{u}_{h}-\widehat{\mathbf{u}}\right) w_{h}=\int_{T}(f-\bar{f}) w_{h}, \quad \forall w_{h} \in W_{h}(T),
\end{array}
$$

where $W_{h}(T)$ is the restriction of $W_{h}$ on $T$ and $\mathbf{V}_{h}^{0}(T)$ is the restriction of $\mathbf{V}_{h}$ on $T$ containing vector fields with zero normal component on $\partial T$. Taking $\mathbf{v}_{h}=\mathbf{u}_{h}-\widehat{\mathbf{u}}$ and $w_{h}=p_{h}-\widehat{p}$ in (4.4), and summing up the resulting equations, we obtain

$$
\int_{T} k^{-1}\left(\mathbf{u}_{h}-\widehat{\mathbf{u}}\right) \cdot\left(\mathbf{u}_{h}-\widehat{\mathbf{u}}\right)=\int_{T}(f-\bar{f})\left(p_{h}-\widehat{p}\right) .
$$


Recall that the Raviart-Thomas elements satisfy the following inf-sup condition [7]:

$$
\left\|w_{h}\right\|_{L^{2}(T)} \preceq \sup _{\mathbf{v}_{h} \in \mathbf{V}_{h}(T)} \frac{\int_{T} \operatorname{div}\left(\mathbf{v}_{h}\right) w_{h}}{\left\|\mathbf{v}_{h}\right\|_{H(\operatorname{div} ; T)}}, \quad \forall w_{h} \in W_{h}(T),
$$

where $\mathbf{V}_{h}(T)$ is the restriction of $\mathbf{V}_{h}$ on $T$. Using the inf-sup condition (4.6) and the error equation (4.4), we have

$$
\left\|p_{h}-\widehat{p}\right\|_{L^{2}(T)} \preceq k_{\min , T}^{-\frac{1}{2}}\left\|\mathbf{u}_{h}-\widehat{\mathbf{u}}\right\|_{k^{-1}, T} .
$$

Finally, by (4.5), we obtain

$$
\left\|\mathbf{u}_{h}-\widehat{\mathbf{u}}\right\|_{k^{-1}, T} \preceq k_{\min , T}^{-\frac{1}{2}}\|f-\bar{f}\|_{L^{2}(T)} .
$$

Also, by (4.4), we have

$$
\left\|\operatorname{div}\left(\mathbf{u}_{h}-\widehat{\mathbf{u}}\right)\right\|_{L^{2}(T)}=\sup _{w_{h} \in W_{h}(T)} \frac{\int_{T} \operatorname{div}\left(\mathbf{u}_{h}-\widehat{\mathbf{u}}\right) w_{h}}{\left\|w_{h}\right\|_{L^{2}(T)}}=\sup _{w_{h} \in W_{h}(T)} \frac{\int_{T}(f-\bar{f}) w_{h}}{\left\|w_{h}\right\|_{L^{2}(T)}} \leq\|f-\bar{f}\|_{L^{2}(T)} .
$$

Collecting results for all coarse-grid elements, we have the desired estimate (4.3).

Now the convergence theorem for the mixed GMsFEM (2.5) is expressed and proved in the following.

Theorem 4.2. Let $\mathbf{u}_{h}$ be the fine-grid solution obtained in (2.3) and $\mathbf{u}_{H}$ be the mixed GMsFEM solution obtained in (2.5). Then, the following estimate holds

$$
\int_{\Omega} k^{-1}\left|\mathbf{u}_{h}-\mathbf{u}_{H}\right|^{2} \preceq \Lambda^{-1} \sum_{i=1}^{N_{t}} a_{i}\left(p_{h}, p_{h}\right)+\max _{T \in \mathcal{T}^{H}}\left(k_{\min , T}^{-1}\right) \sum_{i=1}^{N_{t}}\|f-\bar{f}\|_{L^{2}\left(T_{i}\right)}^{2},
$$

where $\Lambda=\min _{1 \leq i \leq N_{t}} \lambda_{l_{i}+1}^{(i)}$ and $\widehat{\mathbf{u}}$ is the projection of $\mathbf{u}_{h}$ defined in (4.1)-(4.2).

Proof. Subtracting (2.3) by (2.5), and using the fact that $W_{H} \subset W_{h}$, we have

$$
\begin{aligned}
& \int_{\Omega} k^{-1}\left(\mathbf{u}_{h}-\mathbf{u}_{H}\right) \cdot \mathbf{v}_{H}-\int_{\Omega} \operatorname{div}\left(\mathbf{v}_{H}\right)\left(p_{h}-p_{H}\right)=0, \quad \forall \mathbf{v}_{H} \in \mathbf{V}_{h}^{0}, \\
& \int_{\Omega} \operatorname{div}\left(\mathbf{u}_{h}-\mathbf{u}_{H}\right) w_{H}=0, \quad \forall w_{H} \in W_{H} .
\end{aligned}
$$

By (4.4), for each coarse-grid element $T$, we have

$$
\int_{T} \operatorname{div}\left(\mathbf{u}_{h}-\widehat{\mathbf{u}}\right) w_{H}=\int_{T}(f-\bar{f}) w_{H}=0, \quad \forall w_{H} \in W_{H}
$$


by definition of $\bar{f}$. Thus, (4.8) can be written as

$$
\begin{aligned}
\int_{\Omega} k^{-1}\left(\mathbf{u}_{h}-\mathbf{u}_{H}\right) \cdot \mathbf{v}_{H}-\int_{\Omega} \operatorname{div}\left(\mathbf{v}_{H}\right)\left(\widehat{p}-p_{H}\right) & =\int_{\Omega} \operatorname{div}\left(\mathbf{v}_{H}\right)\left(p_{h}-\widehat{p}\right), \quad \forall \mathbf{v}_{H} \in \mathbf{V}_{h}^{0} \\
\int_{\Omega} \operatorname{div}\left(\widehat{\mathbf{u}}-\mathbf{u}_{H}\right) w_{H} & =0, \quad \forall w_{H} \in W_{H}
\end{aligned}
$$

Taking $\mathbf{v}_{H}=\widehat{\mathbf{u}}-\mathbf{u}_{h}$ in (4.9), we obtain

$$
\int_{\Omega} k^{-1}\left(\mathbf{u}_{h}-\mathbf{u}_{H}\right) \cdot\left(\widehat{\mathbf{u}}-\mathbf{u}_{h}\right)=\int_{\Omega} \operatorname{div}\left(\widehat{\mathbf{u}}-\mathbf{u}_{h}\right)\left(p_{h}-\widehat{p}\right)
$$

Note that we can replace $\widehat{p}$ by any $\widetilde{p} \in W_{H}$,

$$
\int_{\Omega} k^{-1}\left(\mathbf{u}_{h}-\mathbf{u}_{H}\right) \cdot\left(\widehat{\mathbf{u}}-\mathbf{u}_{h}\right)=\int_{\Omega} \operatorname{div}\left(\widehat{\mathbf{u}}-\mathbf{u}_{h}\right)\left(p_{h}-\widetilde{p}\right)
$$

So,

$$
\int_{\Omega} k^{-1}\left|\mathbf{u}_{h}-\mathbf{u}_{H}\right|^{2} \preceq \int_{\Omega} k^{-1}\left|\mathbf{u}_{h}-\widehat{\mathbf{u}}\right|^{2}+\left\|\operatorname{div}\left(\widehat{\mathbf{u}}-\mathbf{u}_{h}\right)\right\|_{L^{2}(\Omega)}\left\|p_{h}-\widetilde{p}\right\|_{L^{2}(\Omega)} .
$$

Next, we choose $\widetilde{p}$ to be a linear combination of the modes for the eigenvalue problem (3.7) that corresponds to the eigenvalues $\lambda_{1}^{T_{i}}, \cdots, \lambda_{l_{i}}^{T_{i}}$. Then we have

$\left\|p_{h}-\widetilde{p}\right\|_{L^{2}\left(T_{i}\right)}^{2} \preceq s_{i}\left(p_{h}-\widetilde{p}, p_{h}-\widetilde{p}\right) \preceq \frac{1}{\lambda_{l_{i}+1}^{T_{i}}} a_{i}\left(p_{h}-\widetilde{p}, p_{h}-\widetilde{p}\right)=\frac{1}{\lambda_{l_{i}+1}^{T_{i}}} \int_{T_{i}} k\left|\nabla\left(p_{h}-\widetilde{p}\right)\right|^{2}$

Considering $\int_{T_{i}} k\left|\nabla\left(p_{h}-\widetilde{p}\right)\right|^{2} \preceq \int_{T_{i}} k\left|\nabla p_{h}\right|^{2}$ and collecting the result of Lemma 4.1, (4.12) and (4.13), we obtain the desired result (4.7).

\section{Numerical examples}

In this section, some numerical examples are presented to illustrate the performance of the proposed mixed GMsFEM (2.5) for approximating the subsurface flow problem (2.1). In the following examples, the computational domain is set to be $\Omega=[0,1]^{2}$. The $N \times N$ uniform mesh is chosen to be the coarse grid $\mathcal{T}^{H}$ and the fine grid $\mathcal{T}^{h}$ is the $n \times n$ uniform mesh. The permeability field $k$ we considered here is shown in Figure 1, which represents fractured porous media. The fine-mesh size is chosen to have the same resolution as the permeability field. To shown the performance of the mixed GMsFEM, we define $\left(\mathbf{u}_{f}, p_{f}\right),\left(\mathbf{u}_{s}, p_{s}\right)$, and $\left(\mathbf{u}_{g}, p_{g}\right)$ as the fine-mesh solution, snapshot solution, and mixed GMsFEM solution, respectively. The snapshot solution is the solution of the local problem (3.1) (Case II) and (3.4) (Case III) with all basis functions in the snapshot space selected. For Case I, the snapshot solution is the same as fine-mesh solution. The theoretic result shows that the mixed GMsFEM solution 


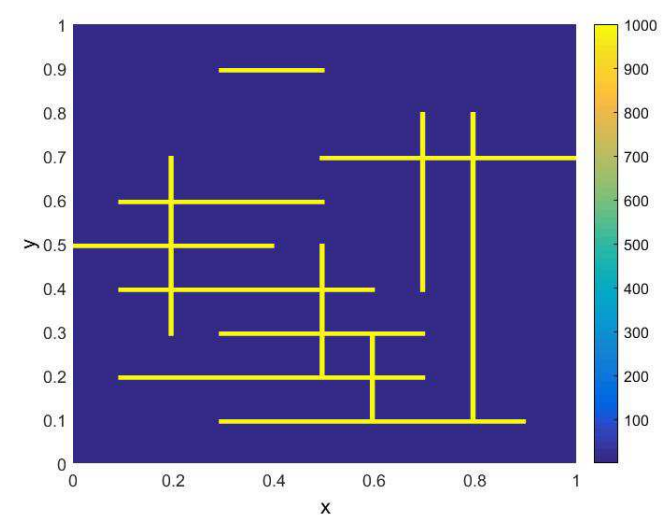

Figure 1: The permeability field of the fractured porous media

contains both coarse-grid and spectral errors and the snapshot solution contains only the coarse-grid error. The following error quantities are defined for the pressure

$$
E_{g f}(p):=\left\|p_{g}-p_{f}\right\|_{L^{2}(\Omega)} /\left\|p_{f}\right\|_{L^{2}(\Omega)}, \quad E_{g s}:=\left\|p_{g}-p_{s}\right\|_{L^{2}(\Omega)} /\left\|p_{s}\right\|_{L^{2}(\Omega)} .
$$

For the velocity, we define

$$
E_{g f}(\mathbf{u}):=\left\|\mathbf{u}_{g}-\mathbf{u}_{f}\right\|_{k^{-1}, \Omega} /\left\|\mathbf{u}_{f}\right\|_{k^{-1}, \Omega}, \quad E_{g s}:=\left\|\mathbf{u}_{g}-\mathbf{u}_{s}\right\|_{k^{-1}, \Omega} /\left\|\mathbf{u}_{s}\right\|_{k^{-1}, \Omega} .
$$

\subsection{Single-phase flow}

In this subsection, we consider the single-phase flow in fractured porous media. In the simulation, the fine-grid size is set to be $n=100$ and the coarse-grid size is $N=10$. The permeability field is illustrated in Figure 1. The permeability is 1000 in the fractures and 1 in the matrix. The homogenous Neumann boundary condition $\frac{\partial p}{\partial \mathbf{n}}=0$ is used on northern and southern boundary. The Dirichlet boundary condition $p=100$ is put on the eastern boundary and $p=0$ is used on western boundary. The numerical results of Case I and Case II are shown in Table 3 and the results of Case III are presented in Table 4. The term "dof per $T$ " means the number of basis functions used for that coarse-grid element. The convergence behaviors of the proposed mixed GMsFEM are shown in Table 3 and Table 4. Since each coarse-grid element is decomposed as $10 \times 10$ fine grids, there are 40 fine-grid boundary edges for each coarse-grid element. The two boundary edges belong to the same corner fine-grid element are considered as a single edge in the local problem, so the number of basis functions in the snapshot space is 36 for Case II and III. From Table 3 and Table 4, we can see that the error decreases as more basis functions are added into the offline space. For Case I, since we use the fine-grid space for pressure $W_{h}$ as the snapshot space $W_{\text {snap }}$, the spectral error is the same as the total error. For Case II, both the total error and spectral error converge to the machine precision, which means the snapshot 
Table 1: Error estimation and convergence of the offline solution for Case I and Case II.

\begin{tabular}{|c|c|c|c|c|c|c|}
\hline & \multicolumn{2}{|c|}{ Case I } & \multicolumn{4}{c|}{ Case II } \\
\hline dof per $T$ & $E_{g f}(p)$ & $E_{g f}(\mathbf{u})$ & $E_{g f}(p)$ & $E_{g f}(\mathbf{u})$ & $E_{g s}(p)$ & $E_{g s}(\mathbf{u})$ \\
\hline 1 & 0.1280 & 2.6333 & 0.1280 & 2.6333 & 0.1280 & 2.6333 \\
3 & 0.0548 & 0.5463 & 0.0533 & 0.5381 & 0.0533 & 0.5381 \\
5 & 0.0189 & 0.3015 & 0.0144 & 0.2736 & 0.0144 & 0.2736 \\
10 & 0.0132 & 0.2580 & 0.0062 & 0.1481 & 0.0062 & 0.1481 \\
15 & 0.0051 & 0.1393 & 0.0029 & 0.1103 & 0.0029 & 0.1103 \\
20 & 0.0041 & 0.1235 & 0.0012 & 0.0498 & 0.0012 & 0.0498 \\
25 & 0.0021 & 0.0700 & $7.96 \mathrm{e}-04$ & 0.0301 & $7.96 \mathrm{e}-04$ & 0.0301 \\
30 & 0.0017 & 0.0604 & $3.36 \mathrm{e}-04$ & 0.0130 & $3.36 \mathrm{e}-04$ & 0.0130 \\
35 & 0.0015 & 0.0538 & $1.41 \mathrm{e}-04$ & 0.0041 & $1.41 \mathrm{e}-04$ & 0.0041 \\
36 & 0.0015 & 0.0534 & $4.13 \mathrm{e}-16$ & $4.25 \mathrm{e}-15$ & $5.25 \mathrm{e}-16$ & $4.53 \mathrm{e}-15$ \\
80 & $1.02 \mathrm{e}-04$ & 0.0034 & - & - & - & - \\
100 & $7.46 \mathrm{e}-16$ & $3.32 \mathrm{e}-15$ & - & - & - & - \\
\hline
\end{tabular}

Table 2: Error estimation and convergence of the offline solution for Case III.

\begin{tabular}{|c|c|c|c|c|}
\hline & \multicolumn{4}{|c|}{ Case III } \\
\hline dof per $T$ & $E_{g f}(p)$ & $E_{g f}(\mathbf{u})$ & $E_{g s}(p)$ & $E_{g s}(\mathbf{u})$ \\
\hline 1 & 0.1280 & 2.6333 & 0.1281 & 2.6330 \\
3 & 0.0556 & 0.5439 & 0.0556 & 0.5439 \\
5 & 0.0150 & 0.2754 & 0.0149 & 0.2752 \\
10 & 0.0068 & 0.1609 & 0.0067 & 0.1607 \\
15 & 0.0029 & 0.1092 & 0.0028 & 0.1089 \\
20 & 0.0014 & 0.0519 & 0.0013 & 0.0513 \\
25 & $7.6503 \mathrm{e}-04$ & 0.0251 & $5.9956 \mathrm{e}-04$ & 0.0237 \\
30 & $5.6132 \mathrm{e}-04$ & 0.0159 & $3.4960 \mathrm{e}-04$ & 0.0136 \\
35 & $4.4009 \mathrm{e}-04$ & 0.0095 & $1.4973 \mathrm{e}-04$ & 0.0045 \\
36 & $4.2434 \mathrm{e}-04$ & 0.0090 & $1.1871 \mathrm{e}-15$ & $1.1077 \mathrm{e}-13$ \\
\hline
\end{tabular}

space is very close to the fine-grid space. For Case III, the spectral error converges to the machine precision.

The reciprocals of the eigenvalues in a particular coarse element for three cases are shown in Figure 2. Please note that the first 0 eigenvalue is not included in figures. It is obvious that there is a rapid decay for the first several eigenvalues and the eigenvalues decrease all the time. This eigenvalue behaviors is corresponding to the error estimations shown in Table 3 and Table 4.

The pressure and velocity solved on fine and coarse meshes are shown in Figure 3 and 4, respectively. The mixed GMsFEM solutions of Case I, II and III are illustrated in Figure 5, 6 and 7 with "dof per $T$ " being 40,25 and 36, respectively. It's obvious that all the cases have a competitive performance of fine-grid solutions. 

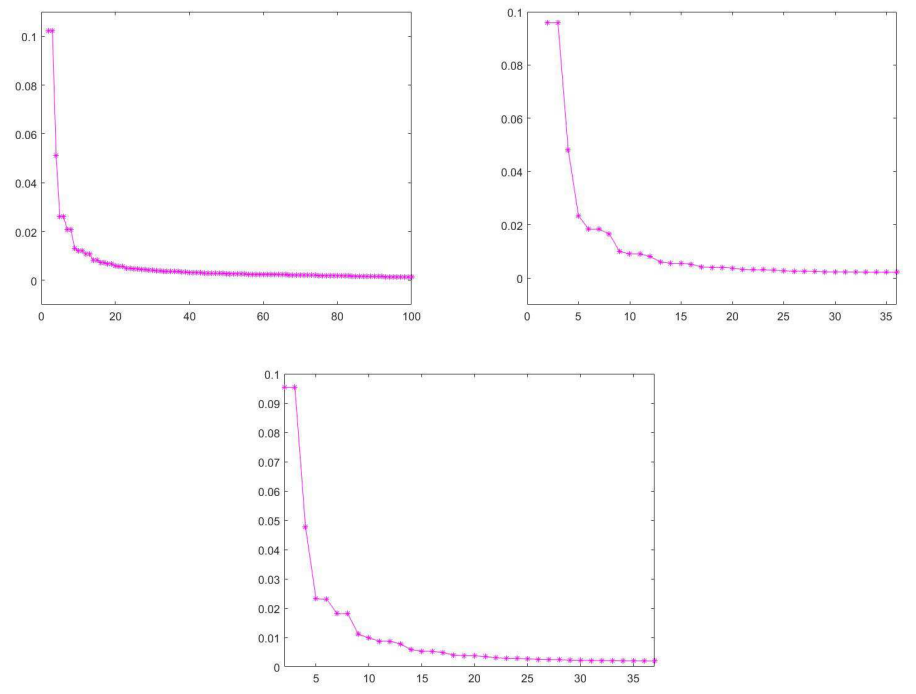

Figure 2: Inverse of eigenvalue behavior for three cases. Top left: Case I. Top right: Case II. Bottom: Case III
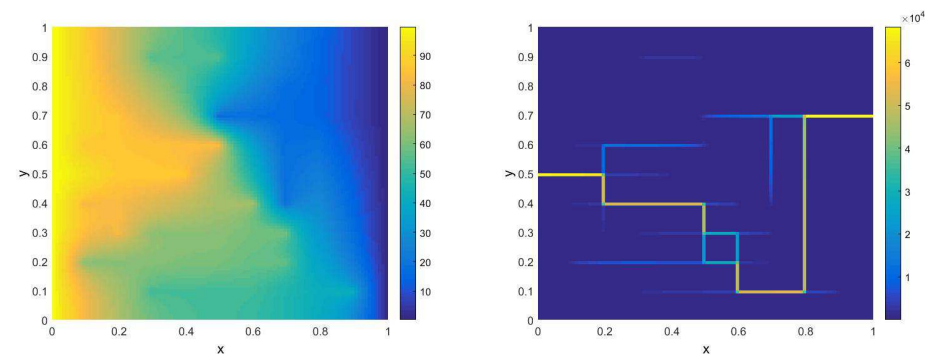

Figure 3: Fine grid solution. left: pressure. right: magnitude of velocity.
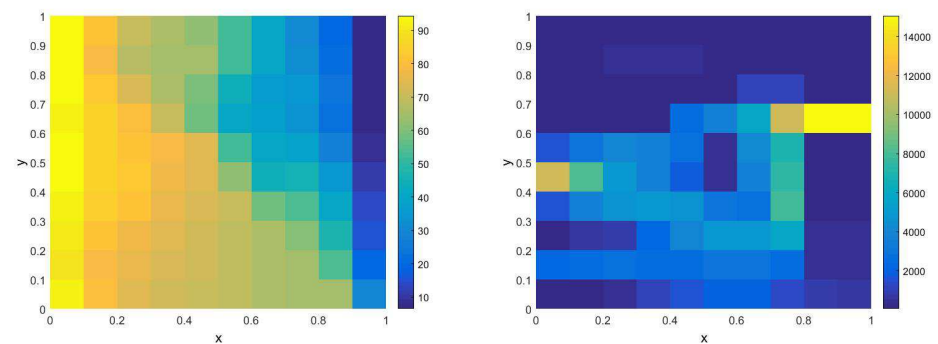

Figure 4: Coarse grid solution. left: pressure. right: magnitude of velocity. 

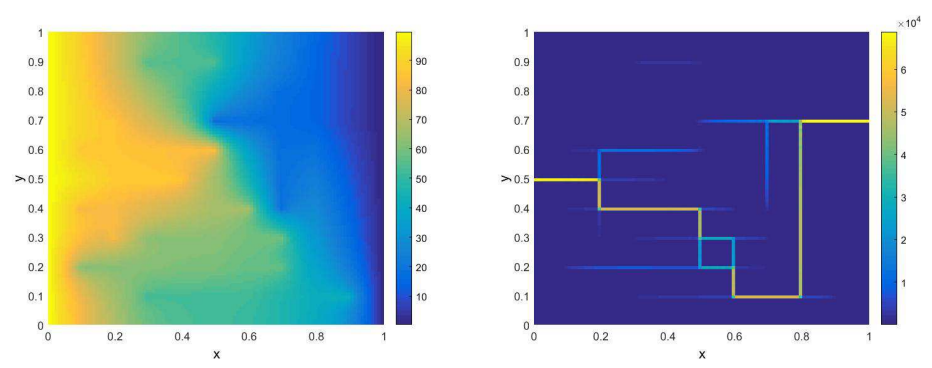

Figure 5: Mixed GMsFEM solution for Case I. left: pressure. right: magnitude of velocity.
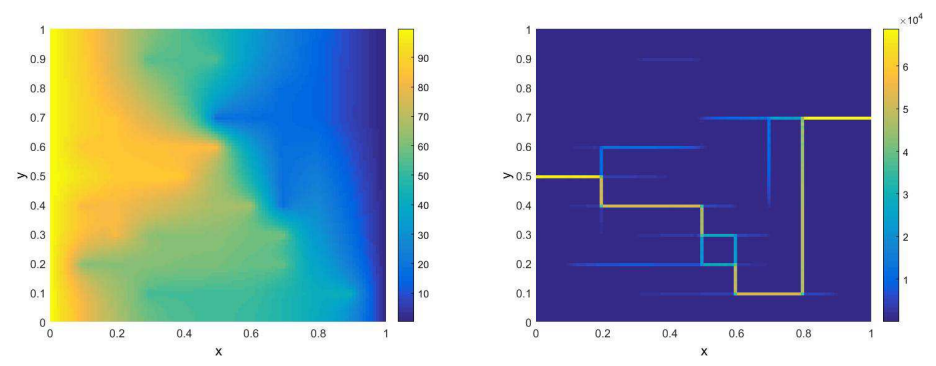

Figure 6: Mixed GMsFEM solution for Case II. left: pressure. right: magnitude of velocity.
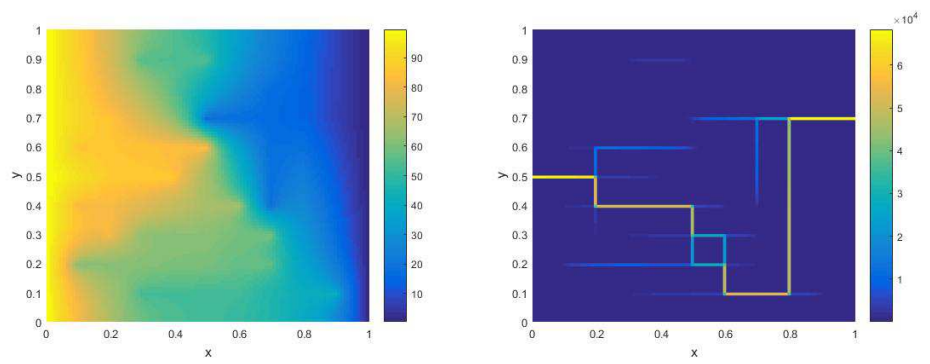

Figure 7: Mixed GMsFEM solution for Case III. left: pressure. right: magnitude of velocity. 


\subsection{Single-phase flow and transport problems}

In this subsection, we employ the proposed mixed GMsFEM to simulate the singlephase flow and transport problems. Specifically, the following problem with homogenous Neumann boundary condition is considered:

$$
\begin{aligned}
k^{-1} \mathbf{u}+\nabla p=0 & \text { in } \quad \Omega, \\
\nabla \cdot \mathbf{u}=f & \text { in } \quad \Omega, \\
\mathbf{u} \cdot \mathbf{n}=0 & \text { on } \quad \partial \Omega,
\end{aligned}
$$

together with the saturation equation

$$
S_{t}+\mathbf{u} \cdot \nabla S=r,
$$

where $S$ represents the saturation and $r$ is the source term. $f$ is zero except the bottom left and top right fine-grid elements, where $f$ is 1 and -1 , respectively. $r$ is zero except the bottom left fine-grid element, where $r$ is 1 . Time step size is 0.01 . We use the mixed GMsFEM to solve the flow equation and the saturation equation is directly solved on fine mesh explicitly.

Since the velocity solution of mixed GMsFEM preserves the mass conservation property only in the coarse mesh but not in the fine mesh, we need to do a postprocessing procedure to make it compatible with the transport in the fine mesh [36]. We illustrate the postprocessing procedure as following. First we calculate the residual on each coarse-grid element

$$
R_{T}\left(\mathbf{u}_{H}\right)=\sum_{i=1}^{n}\left(\int_{t_{i}} f-\int_{\partial t_{i}} \mathbf{u}_{H} \cdot \mathbf{n}\right),
$$

where $\mathbf{u}_{H}$ is the velocity solution of mixed GMsFEM; $t_{i}$ is a fine-grid element contained in coarse-grid element $T$, and $n$ is the number of fine-grid elements contained in coarse-grid element $T . f$ is the source term in flow equation.

Then solving the following local problem, we get a correction of velocity

$$
\begin{aligned}
& \nabla \cdot \mathbf{u}^{c}=R_{T}\left(\mathbf{u}_{H}\right) \quad \text { in } \quad T \\
& \mathbf{u}^{c}=-\nabla \Phi \quad \text { in } \quad T
\end{aligned}
$$

with homogenous boundary condition

$$
\mathbf{u}^{c} \cdot \mathbf{n}=0 \quad \text { on } \quad \partial T,
$$

where $\mathbf{u}^{c}$ is a correction of velocity field and $\Phi$ is a supplementary variable. The corrected solution of velocity $\widehat{\mathbf{u}_{H}}=\mathbf{u}_{H}+\mathbf{u}^{c}$ is locally conservative in fine mesh, i.e. $R_{T}\left(\widehat{\mathbf{u}_{H}}\right)$ is of machine precision. Then $\widehat{\mathbf{u}_{H}}$ is used for solving transport equation explicitly. Let $\Delta t$ be the time step size, and $S_{i}^{n}$ be the value of $S$ on the fine grid $t_{i}$. 

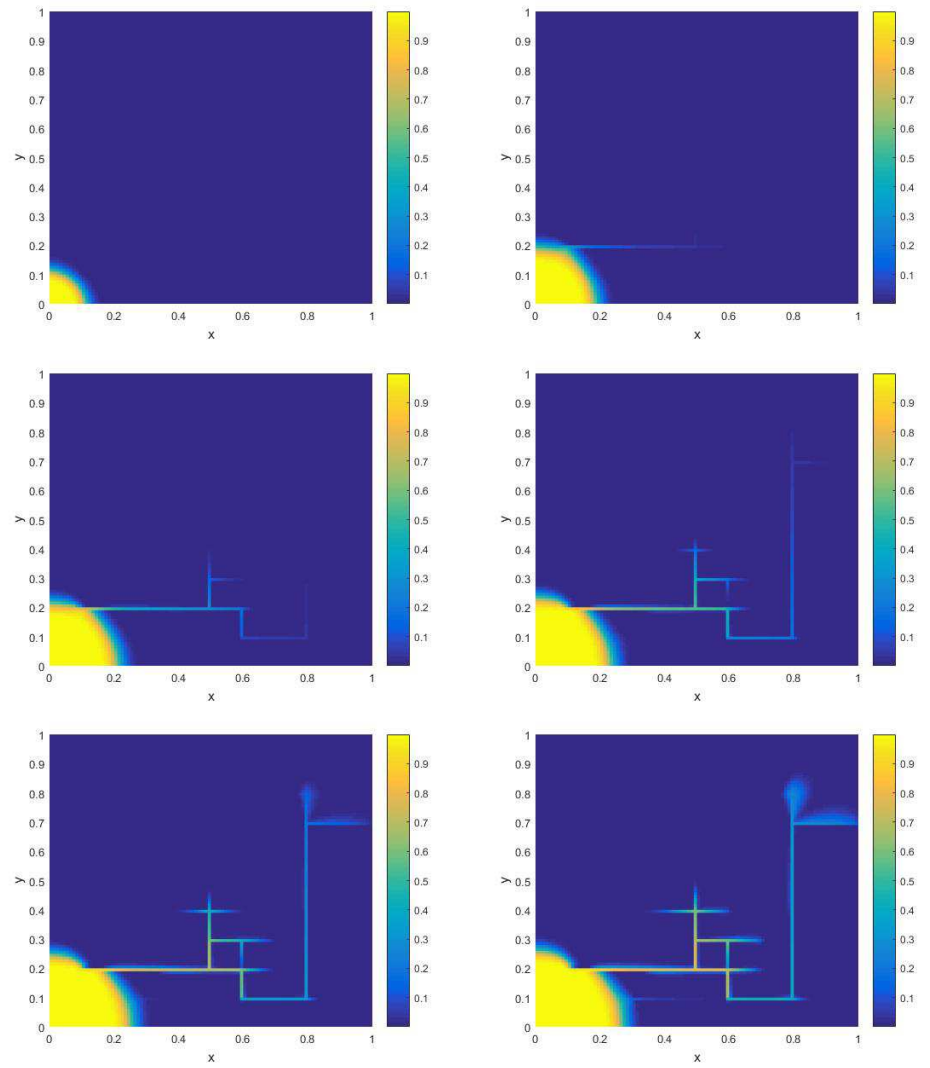

Figure 8: Snapshots of saturation $S$ on the fine mesh calculated by time step $\Delta t=0.01$ at time $t=$ $100,300,400,500,600,700$.

The saturation $S_{i}^{n}$ is updated by the following discretized equation explicitly.

$$
\left|t_{i}\right| \frac{S_{i}^{n+1}-S_{i}^{n}}{\Delta t}+\int_{\partial t_{i}} \hat{S}^{n}\left(\widehat{\mathbf{u}_{H}} \cdot \mathbf{n}\right)=r_{i}\left|t_{i}\right|
$$

where $\hat{S}^{n}\left(\widehat{\mathbf{u}_{H}} \cdot \mathbf{n}\right)$ is the upwind flux; $\left|t_{i}\right|$ is the area of the fine element $t_{i}$ and $r_{i}$ is the averaged value of $r$ on $t_{i}$.

We present the solutions of saturation on fine mesh in Figure 8 as a reference. The saturations for Case I, II and III are shown in Figure 9, Figure 10 and Figure 11, respectively. Obviously, all the cases we presented have a competitive performance of fine-grid solutions.

\subsection{Two-phase flow and transport problems}

In this subsection, the performance of the proposed mixed GMsFEM for two-phase flow and transport problems is reported. The following problem with homogenous 

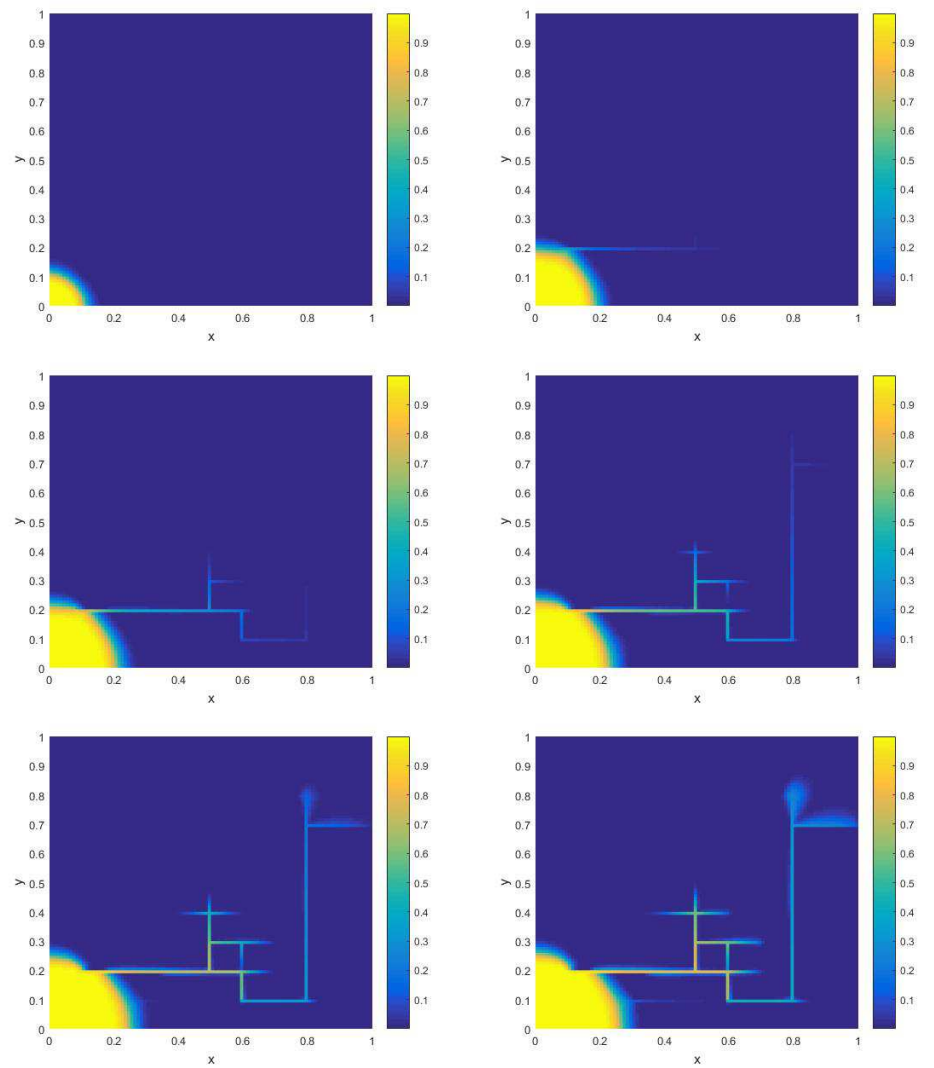

Figure 9: Snapshots of saturation $S$ for Case I calculated by time step $\Delta t=0.01$ at time $t=$ 100, 300, 400, 500, 600, 700 with "dof per $T$ " being 40. 

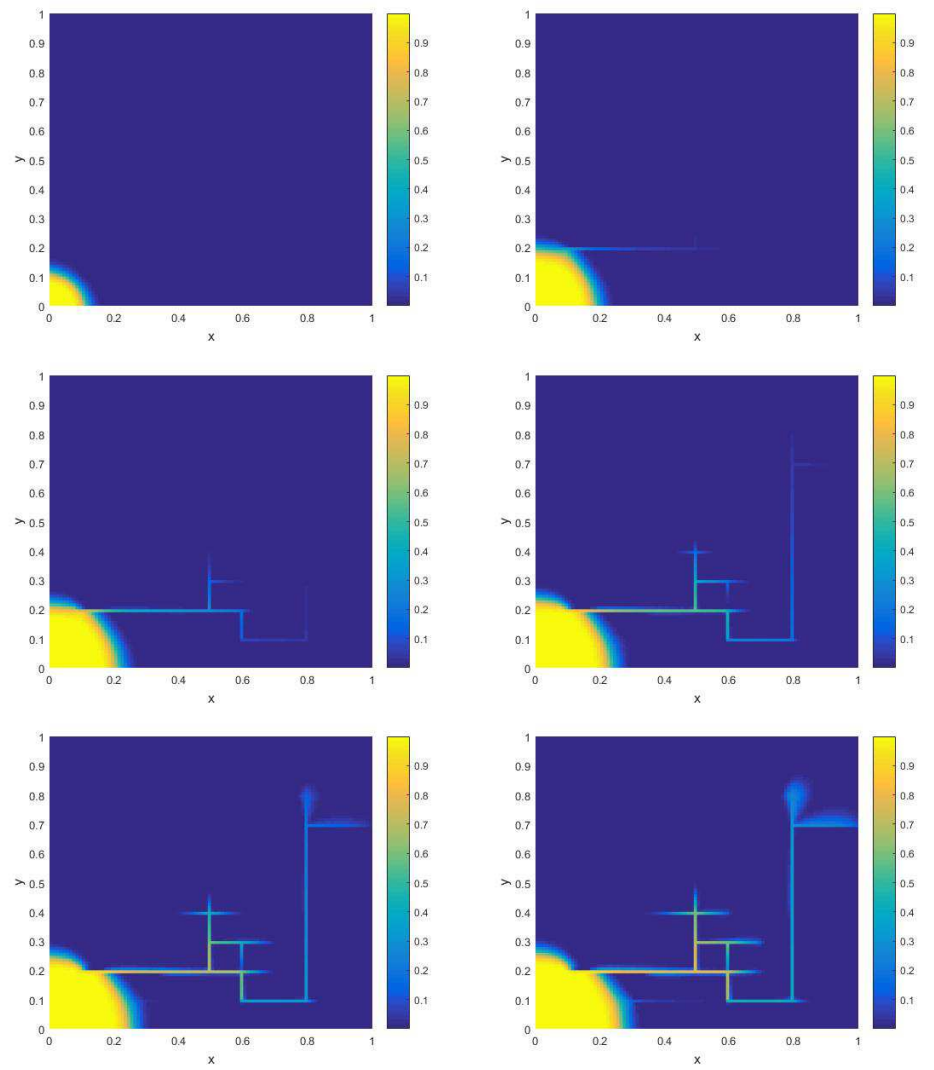

Figure 10: Snapshots of saturation $S$ for Case II calculated by time step $\Delta t=0.01$ at time $t=$ $100,300,400,500,600,700$ with "dof per $T$ " being 25 . 

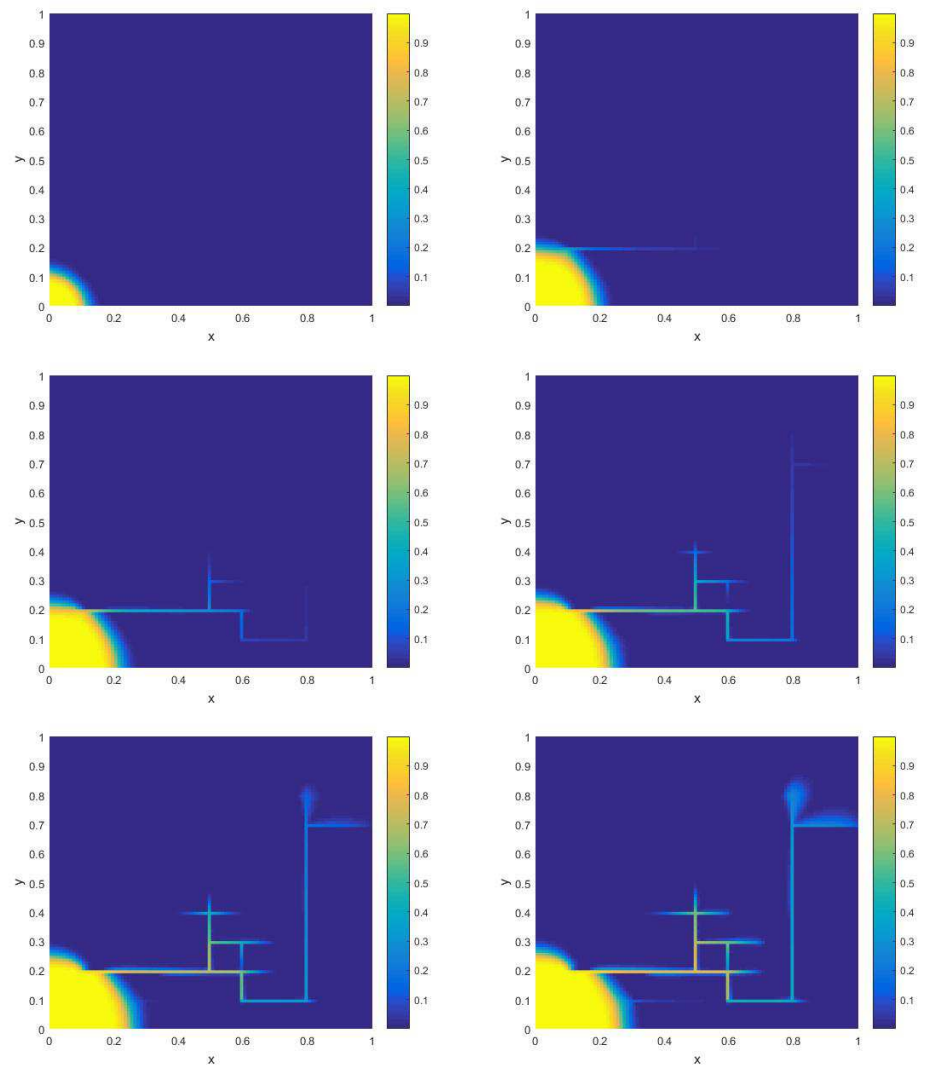

Figure 11: Snapshots of saturation $S$ for Case III calculated by time step $\Delta t=0.01$ at time $t=$ $100,300,400,500,600,700$ with "dof per $T$ " being 25 . 
Neumann boundary condition is considered:

$$
\begin{aligned}
-\eta(S) k \nabla p=\mathbf{u} & \text { in } \quad \Omega, \\
\nabla \cdot \mathbf{u}=f & \text { in } \quad \Omega, \\
\mathbf{u} \cdot \mathbf{n}=0 & \text { on } \quad \partial \Omega,
\end{aligned}
$$

where

$$
\eta(S)=\frac{k_{r w}(S)}{\mu_{w}}+\frac{k_{r o}(S)}{\mu_{o}}
$$

is the total mobility and

$$
k_{r w}(S)=S^{2}, \quad k_{r o}(S)=(1-S)^{2}, \quad \mu_{w}=1, \quad \mu_{o}=5 .
$$

The transport equation is

$$
S_{t}+\mathbf{u} \cdot \nabla F(S)=r,
$$

where $S$ represents the saturation; $r$ is the source term and

$$
F(S)=\frac{k_{r w}(S) / \mu_{w}}{k_{r w}(S) / \mu_{w}+k_{r o}(S) / \mu_{o}} .
$$

Similar to the previous case, we employ a postprocessing procedure to make the velocity solution of mixed GMsFEM compatible with the transport in the fine mesh. Then the corrected velocity solution $\widehat{\mathbf{u}_{H}}$ is used for solving transport equation explicitly.

$$
\left|t_{i}\right| \frac{S_{i}^{n+1}-S_{i}^{n}}{\Delta t}+\int_{\partial t_{i}} F\left(\hat{S}^{n}\right)\left(\widehat{\mathbf{u}_{H}} \cdot \mathbf{n}\right)=r_{i}\left|t_{i}\right|,
$$

where the same source terms $f$ and $r$ as in the single-phase flow case are used.

In the single-phase flow case, we only solve the flow equations once and the velocity field doesn't change in the whole simulation. However, the velocity field needs to be updated for the two-phase flow case. We carefully set the time step sizes to save the computational cost. Since the pressure changes less rapidly in time than the saturation in porous media and the constraint on time steps is primarily used in the explicit calculation of saturation. Furthermore, the multiscale pressure space changes more slowly than the pressure solution itself. All these reasons motivate us to set the time step sizes as following.

$$
\begin{aligned}
\Delta t_{p} & =N_{p} \Delta t_{s}, \\
\Delta t_{g} & =N_{g} \Delta t_{p} .
\end{aligned}
$$

At time step $\Delta t_{g}$ we solve the local cell problem to obtain the snapshot space and then solve the spectral problem to construct the multiscale pressure space. At time step $\Delta t_{p}$ we solve the flow equations in the multiscale space and correct the velocity 

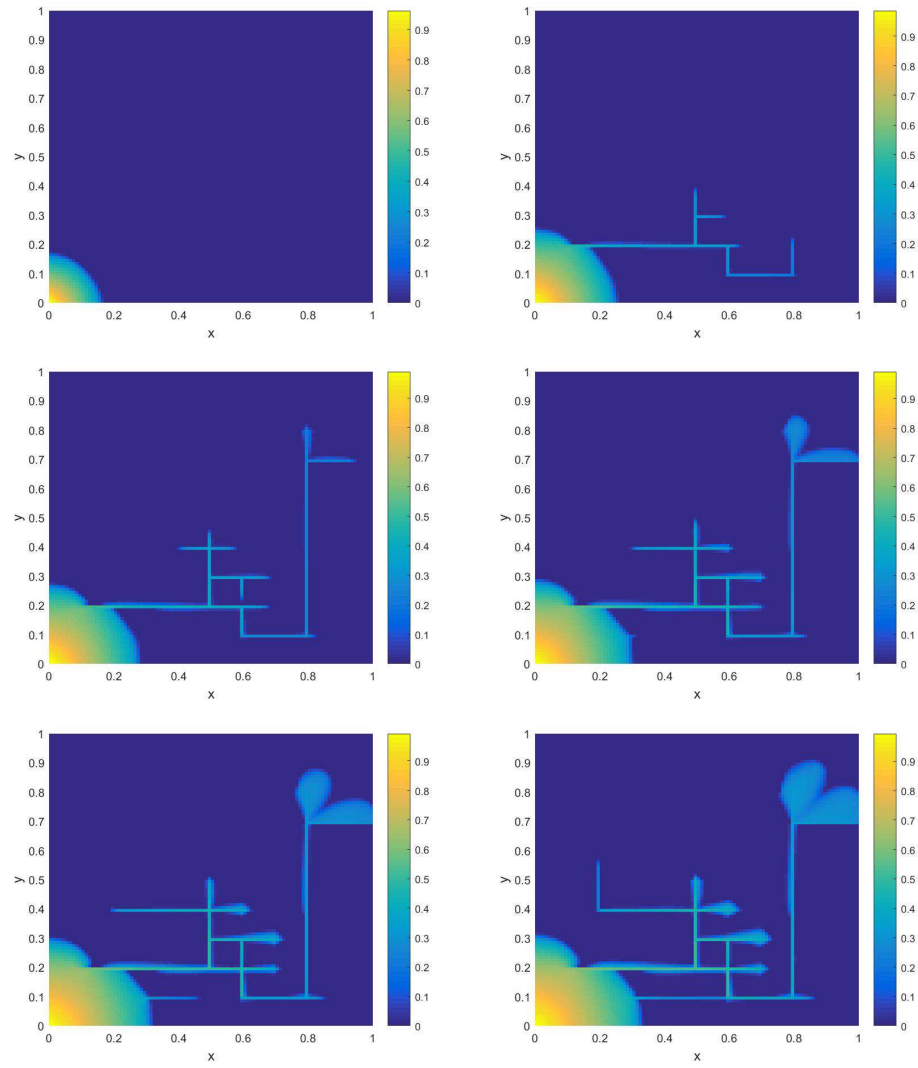

Figure 12: Snapshots of saturation $S$ on the fine mesh calculated by time step $\Delta t_{s}=0.01, N_{p}=100$ and $N_{g}=1$ at time $t=100,300,400,500,600,700$.

solution. At every times step $\Delta t_{s}$ we employ the corrected velocity solution to update the saturation explicitly. $N_{p}$ and $N_{g}$ are positive integers to be chosen. So, totally we have three levels of time steps, the fine time step $\Delta t_{s}$, the medium time step $\Delta t_{p}$ and the coarse time step $\Delta t_{g}$. This set of time steps will save the computational time greatly.

We present the solutions of saturation on fine mesh in Figure 12 as a reference. The saturations for Case I and II are shown in Figure 13 and Figure 14, respectively. For Case I, we don't need to solve the local cell problem on the coarse time step $\Delta t_{g}$, so we set $N_{p}=100$ and $N_{g}=1 . N_{p}=10$ and $N_{g}=10$ are chosen for the Case II. It is obvious that both cases give a competitive performance of fine-grid solutions.

\subsection{Comparison between the proposed method and the previous method}

The convergence results of the proposed method have the similar form of our previous work [14]. This is because both theoretical conclusions are obtained by the spectral analysis. The error bounds depend on the reciprocal of the smallest unused eigenvalue. 

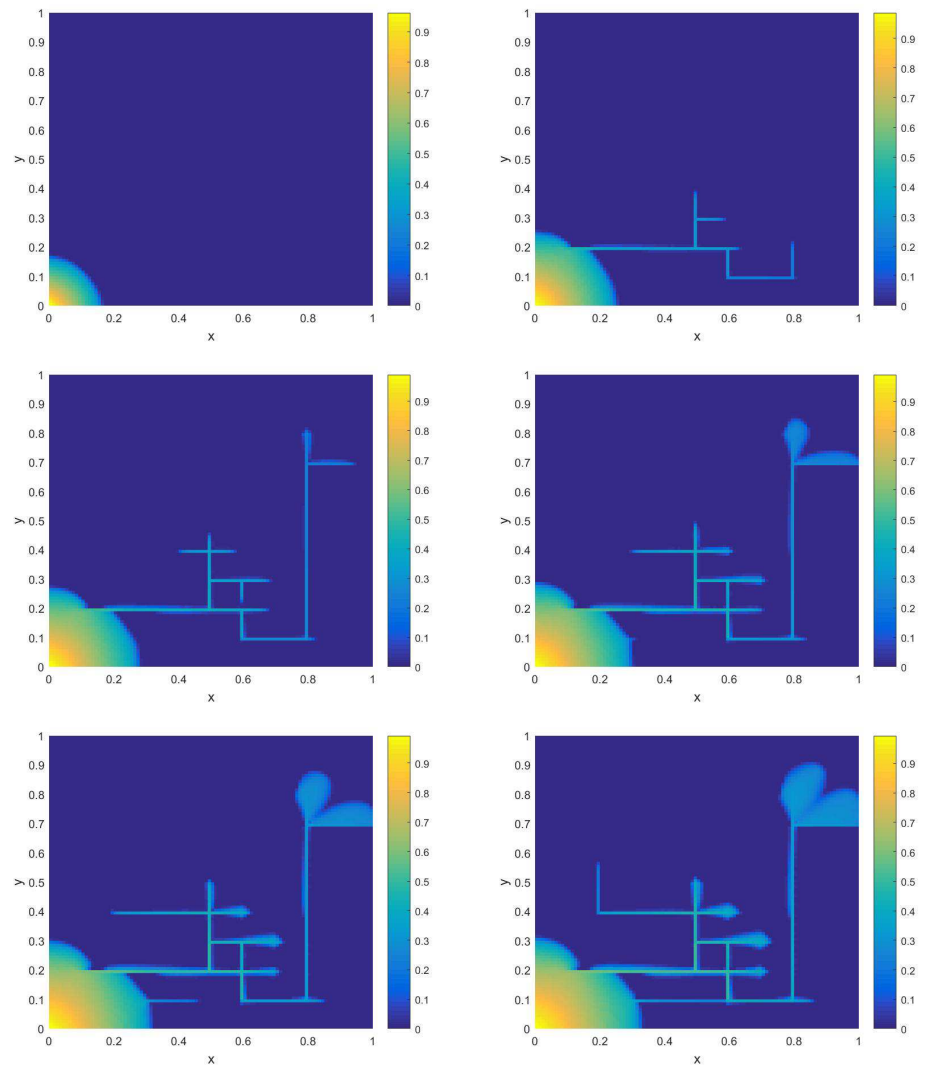

Figure 13: Snapshots of saturation $S$ for Case I calculated by time step $\Delta t_{s}=0.01, N_{p}=100$ and $N_{g}=1$ at time $t=100,300,400,500,600,700$ with "dof per $T$ " being 40 . 

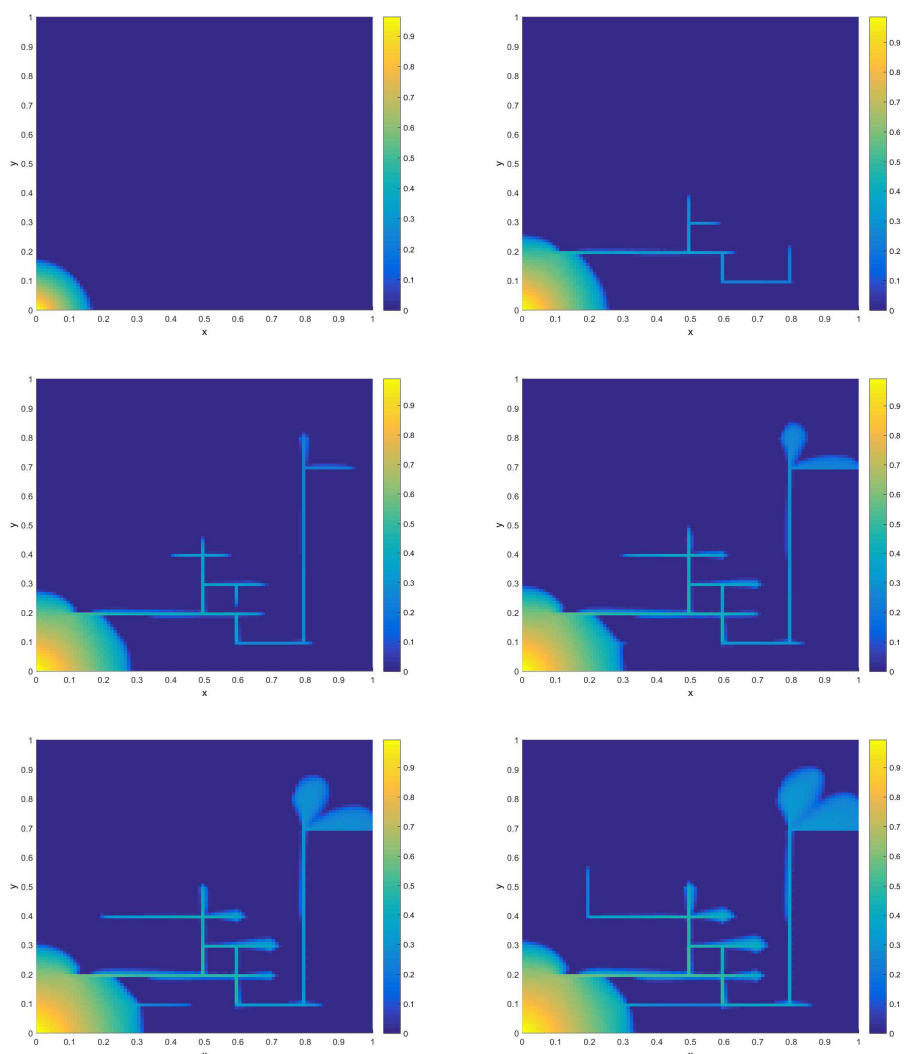

Figure 14: Snapshots of saturation $S$ for Case II calculated by time step $\Delta t_{s}=0.01, N_{p}=10$ and $N_{g}=10$ at time $t=100,300,400,500,600,700$ with "dof per $T$ " being 25 . 


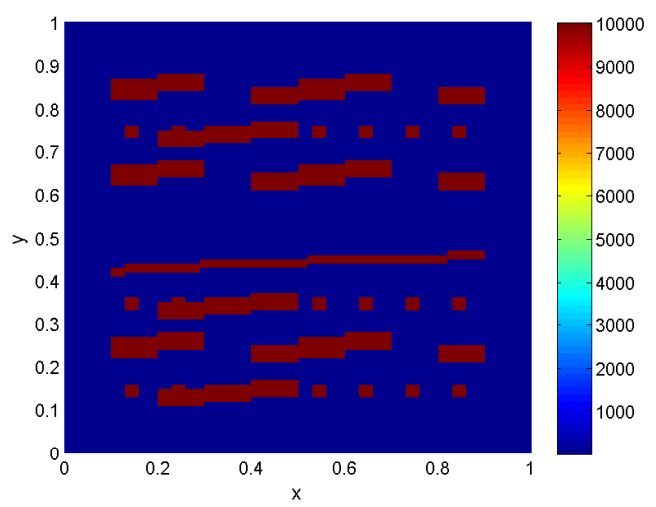

Figure 15: The permeability field $\kappa_{1}$ of the porous media [14].

However, since both the pressure and velocity are approximated in bigger spaces, the new method enjoys better accuracy than the previous method, especially for the pressure. This can be illustrated by the following numerical comparisons. We resimulate the numerical example 1 "Single-phase flow" in [14] and compare the error bounds of both algorithms.

The permeability field $\kappa_{1}$ is shown in Figure 15. For the ease of comparison, we use two different coarse-mesh sizes with $N=10$ and $N=20$, called case 1 and case 2 , which is same as the previous work [14]. Notice that cases 1 and 2 decompose each coarse-grid block as $20 \times 20$ and $10 \times 10$ grids, respectively. The error bounds of both methods for the permeability field $\kappa_{1}$ are shown in Table 3 and 4 for case 1 and case 2 , respectively. For the previous method, the multiscale base of velocity on the coarse edge and the piecewise constant function in the coarse grid for pressure are used as freedoms. The new method employs the multiscale base of pressure in the coarse grid as unknows. As a consequence, "dof per $T$ " $=2 \times$ "dof per $E$ " +1 , where "dof per $T$ " is the number of basis functions used for that coarse-grid element $T$ and "dof per $E$ " is the number of basis functions used for that coarse edge $E$. From these tables, we see clearly the convergence of both methods when basis functions are added to the offline space. The new method enjoys better accuracy than the previous method, especially for the pressure.

\section{6. conclusions}

In this paper, we propose a frame work of the mixed generalized multiscale finite element method for solving subsurface flow problem in heterogeneous media. The main contribution of this work lies on constructing a systematic enrichment for multiscale basis functions for the pressure. We raise three types of local cell problems to construct the snapshot space. We describe a spectral decomposition in the snapshot space motivated by the analysis to further reduce the dimension of the space that is used to approximate the pressure. The convergence of the proposed method is analysed. The 
Table 3: Comparisons of error bounds between the new method and our previous method, $n=200, N=$ 10.

\begin{tabular}{|c|c|c|c|c|c|}
\hline & \multicolumn{2}{|c|}{ The new method } & \multicolumn{2}{c|}{ The previous method } & \\
\hline dof per $T$ & $E_{g f}(p)$ & $E_{g f}(\mathbf{u})$ & $E_{g f}(p)$ & $E_{g f}(\mathbf{u})$ & dof per $E$ \\
\hline 1 & 0.0851 & 3.1963 & - & - & - \\
3 & 0.0630 & 0.6651 & 0.0903 & 0.1331 & 1 \\
7 & 0.0020 & 0.0371 & 0.0896 & 0.0569 & 3 \\
11 & $6.9365 \mathrm{e}-04$ & 0.0192 & 0.0898 & 0.0308 & 5 \\
15 & $3.8857 \mathrm{e}-04$ & 0.0136 & 0.0898 & 0.0236 & 7 \\
19 & $2.5469 \mathrm{e}-04$ & 0.0106 & 0.0898 & 0.0210 & 9 \\
23 & $1.6394 \mathrm{e}-04$ & 0.0080 & 0.0898 & 0.0208 & 11 \\
41 & $3.5303 \mathrm{e}-05$ & 0.0029 & 0.0898 & 0.0208 & 20 \\
\hline
\end{tabular}

Table 4: Comparisons of error bounds between the new method and our previous method, $n=200, N=$ 20.

\begin{tabular}{|c|c|c|c|c|c|}
\hline & \multicolumn{2}{|c|}{ The new method } & \multicolumn{2}{c|}{ The previous method } & \\
\hline dof per $T$ & $E_{g f}(p)$ & $E_{g f}(\mathbf{u})$ & $E_{g f}(p)$ & $E_{g f}(\mathbf{u})$ & dof per $E$ \\
\hline 1 & 0.0477 & 2.4040 & - & - & - \\
3 & 0.0395 & 0.2734 & 0.0601 & 0.1788 & 1 \\
5 & $8.7752 \mathrm{e}-04$ & 0.0363 & 0.0486 & 0.0460 & 2 \\
7 & $4.9249 \mathrm{e}-04$ & 0.0164 & 0.0486 & 0.0251 & 3 \\
9 & $2.5304 \mathrm{e}-04$ & 0.0107 & 0.0486 & 0.0115 & 4 \\
11 & $1.6364 \mathrm{e}-04$ & 0.0081 & 0.0486 & 0.0054 & 5 \\
21 & $3.7923 \mathrm{e}-05$ & 0.0030 & 0.0486 & 0.0054 & 10 \\
\hline
\end{tabular}


resulting linear system of our method is easy to solve because of the good property of velocity matrix. Numerical examples including single-phase and two-phase flow with transport problems are tested to verify the performance of our proposed method.

[1] J.E. Aarnes. On the use of a mixed multiscale finite element method for greater flexibility and increased speed or improved accuracy in reservoir simulation. SIAM J. Multiscale Modeling and Simulation, 2:421-439, 2004.

[2] J.E. Aarnes, Y. Efendiev, and L. Jiang. Analysis of multiscale finite element methods using global information for two-phase flow simulations. SIAM J. Multiscale Modeling and Simulation, 7:2177-2193, 2008.

[3] J.E. Aarnes, S. Krogstad, and K.-A. Lie. A hierarchical multiscale method for two-phase flow based upon mixed finite elements and nonuniform grids. SIAM J. Multiscale Modeling and Simulation, 5(2):337-363, 2006.

[4] T. Arbogast, G. Pencheva, M.F. Wheeler, and I. Yotov. A multiscale mortar mixed finite element method. SIAM J. Multiscale Modeling and Simulation, 6(1):319_ 346, 2007.

[5] Todd Arbogast, Mary F Wheeler, and Ivan Yotov. Mixed finite elements for elliptic problems with tensor coefficients as cell-centered finite differences. SIAM Journal on Numerical Analysis, 34(2):828-852, 1997.

[6] J.W. Barker and S. Thibeau. A critical review of the use of pseudorelative permeabilities for upscaling. SPE Reservoir Eng., 12:138-143, 1997.

[7] F. Brezzi and M. Fortin. Mixed and hybrid finite element methods, volume 15 of Springer Series in Computational Mathematics. Springer-Verlag, New York, 1991.

[8] L. Bush and V. Ginting. On the application of the continuous Galerkin finite element method for conservation problems. SIAM Journal on Scientific Computing, 35(6):A2953-A2975, 2013.

[9] H. Y. Chan, E. T. Chung, and Y. Efendiev. Adaptive mixed gmsfem for flows in heterogeneous media. Numerical Mathematics: Theory, Methods and Applications, 9(4):497-527, 2016.

[10] F. Chen, E. Chung, and L. Jiang. Least-squares mixed generalized multiscale finite element method. Computer Methods in Applied Mechanics and Engineering, 311:764-787, 2016.

[11] Y. Chen, L. Durlofsky, M. Gerritsen, and X. Wen. A coupled local-global upscaling approach for simulating flow in highly heterogeneous formations. Advances in Water Resources, 26:1041-1060, 2003. 
[12] Z. Chen and T. Y. Hou. A mixed multiscale finite element method for elliptic problems with oscillating coefficients. Mathematics of Computation, 72(242):541-576, 2003.

[13] E. Chung, Y. Efendiev, and T. Y. Hou. Adaptive multiscale model reduction with generalized multiscale finite element methods. Journal of Computational Physics, 320:69-95, 2016.

[14] E. T. Chung, Y. Efendiev, and C. S. Lee. Mixed generalized multiscale finite element methods and applications. Multiscale Modeling \& Simulation, 13(1):338366, 2015.

[15] E. T. Chung, S. Fu, and Y. Yang. An enriched multiscale mortar space for high contrast flow problems. arXiv preprint arXiv:1609.02610, 2016.

[16] E. T. Chung, W. T. Leung, and M. Vasilyeva. Mixed gmsfem for second order elliptic problem in perforated domains. Journal of Computational and Applied Mathematics, 304:84-99, 2016.

[17] D. Cortinovis and P. Jenny. Iterative Galerkin-enriched multiscale finite-volume method. Journal of Computational Physics, 277:248-267, 2014.

[18] L.J. Durlofsky. Numerical calculation of equivalent grid block permeability tensors for heterogeneous porous media. Water Resour. Res., 27:699-708, 1991.

[19] L.J. Durlofsky. Coarse scale models of two-phase flow in heterogeneous reservoirs: Volume averaged equations and their relation to existing upscaling techniques. Comp. Geosciences, 2:73-92, 1998.

[20] Y. Efendiev, J. Galvis, and T. Hou. Generalized multiscale finite element methods. Journal of Computational Physics, 251:116-135, 2013.

[21] Y. Efendiev and T. Hou. Multiscale Finite Element Methods: Theory and Applications. Springer, 2009.

[22] Y. Efendiev, T. Hou, and X.H. Wu. Convergence of a nonconforming multiscale finite element method. SIAM J. Numer. Anal., 37:888-910, 2000.

[23] Y. Efendiev, O. Iliev, and P. S. Vassilevski. Mini-workshop: Numerical upscaling for media with deterministic and stochastic heterogeneity. Oberwolfach Reports, 10(1):393-431, 2013.

[24] H. Hajibeygi, D. Kavounis, and P. Jenny. A hierarchical fracture model for the iterative multiscale finite volume method. Journal of Computational Physics, 230(4):8729-8743, 2011.

[25] T. Hou and X.H. Wu. A multiscale finite element method for elliptic problems in composite materials and porous media. J. Comput. Phys., 134:169-189, 1997. 
[26] T.J.R. Hughes. Multiscale phenomena: Green's functions, the dirichlet-toneumann formulation, subgrid scale models, bubbles and the origins of stabilized methods. Comput. Methods Appl. Mech Engrg., 127:387-401, 1995.

[27] P. Jenny, S.H. Lee, and H. Tchelepi. Multi-scale finite volume method for elliptic problems in subsurface flow simulation. J. Comput. Phys., 187:47-67, 2003.

[28] J. Kou, S. Sun, and X. Wang. Linearly decoupled energy-stable numerical methods for multi-component two-phase compressible flow. SIAM Journal on Numerical Analysis, 56(6):3219-3248, 2018.

[29] K-A Lie, O Møyner, JR Natvig, et al. A feature-enriched multiscale method for simulating complex geomodels. In SPE Reservoir Simulation Conference. Society of Petroleum Engineers, 2017.

[30] I. Lunati and P. Jenny. Multi-scale finite-volume method for highly heterogeneous porous media with shale layers. In Proceedings of the 9th European Conference on the Mathematics of Oil Recovery (ECMOR), Cannes, France, 2004.

[31] L. H. Odsæter, M. F. Wheeler, T. Kvamsdal, and M. G. Larson. Postprocessing of non-conservative flux for compatibility with transport in heterogeneous media. Computer Methods in Applied Mechanics and Engineering, 315:799-830, 2017.

[32] M. Peszyńska. Mortar adaptivity in mixed methods for flow in porous media. Int. J. Numer. Anal. Model., 2(3):241-282, 2005.

[33] M. Peszyńska, M. Wheeler, and I. Yotov. Mortar upscaling for multiphase flow in porous media. Comput. Geosci., 6(1):73-100, 2002.

[34] Thomas F Russell and Mary Fanett Wheeler. Finite element and finite difference methods for continuous flows in porous media. In The mathematics of reservoir simulation, pages 35-106. SIAM, 1983.

[35] S. Sun. Darcy-scale phase equilibrium modeling with gravity and capillarity. Journal of Computational Physics, 399:108908, 2019.

[36] Shuyu Sun and Mary F Wheeler. Projections of velocity data for the compatibility with transport. Computer Methods in Applied Mechanics and Engineering, 195(7-8):653-673, 2006.

[37] X.H. Wu, Y. Efendiev, and T.Y. Hou. Analysis of upscaling absolute permeability. Discrete and Continuous Dynamical Systems, Series B., 2:158-204, 2002.

[38] H. Yang, S. Sun, Y. Li, and C. Yang. A fully implicit constraint-preserving simulator for the black oil model of petroleum reservoirs. Journal of Computational Physics, 396:347-363, 2019.

[39] G. Zhu, J. Kou, B. Yao, Y. Wu, J. Yao, and S. Sun. Thermodynamically consistent modelling of two-phase flows with moving contact line and soluble surfactants. Journal of Fluid Mechanics, 879(25):327-359, 2019. 